\title{
Micro-fabrication of high-thickness spiral inductors for the remote powering of implantable biosensors
}

\author{
Jacopo Olivo*, Sandro Carrara, Giovanni De Micheli \\ Integrated Circuits Laboratory, EPFL - École Polytechnique Fédérale de Lausanne, 1015 Lausanne, Switzerland
}

\section{A R T I C L E I N F O}

\section{Article history:}

Received 5 April 2013

Received in revised form 17 July 2013

Accepted 23 July 2013

Available online 3 August 2013

\section{Keywords:}

High-thickness inductors

Inductive link

Remote powering

Implantable biosensors

Ordyl

\begin{abstract}
A B S T R A C T
A micro-fabrication process is proposed to realize high-thickness spiral inductors for the remote powering of implantable biosensors through inductive link. The process is suitable for different substrates, such as silicon and Pyrex, and enables the fabrication of the receiving inductor directly on the implantable system. The use of Ordyl Alpha960 is explored to achieve high-thickness structures. Ordyl is a dry film, negative photoresist that enables high-thickness mold (starting from $60 \mu \mathrm{m}$ ) with a single-layer deposition. Copper spiral inductors with a trace thickness of $60 \mu \mathrm{m}$ are fabricated on silicon and tested. These inductors can receive up to $8.7 \mathrm{~mW}$, with a link efficiency of $25 \%$, over a distance of $6 \mathrm{~mm}$ from the transmitter. Tested within a real setup, these inductors enable bidirectional data communication with the external transmitter. Downlink communication (ASK) is successfully tested at $100 \mathrm{kbps}$. Uplink communication (LSK) is successfully tested at $66.6 \mathrm{kbps}$.
\end{abstract}

(c) 2013 Elsevier B.V. All rights reserved.

\section{Introduction}

The research field of implantable biosensors has been attracting the attention of academia and industry for the past two decades. These devices enable to constantly monitor the human body and its metabolism from inside, thus paving the way to personalized medicine and point-of-care therapies.

Batteries are usually a bottleneck in the miniaturization process of implantable biosensors [1]. To overcome this issue, remote powering through inductive link is often proposed as a solution. In such a technique, near-field magnetic induction is used to transfer power wirelessly through the body tissues. An alternate current, flowing through an external inductor (transmitter) induces an alternate current into one or more implanted inductors (receiver). Inductive link can also be used to enable bidirectional data communication between the implanted devices and the external system. Downlink communication (from the external transmitter to the implanted device) can be achieved by modulating the power carrier generated by the transmitter. When no high data-rates are required, amplitude modulation (Amplitude Shift Keying ASK) is generally preferred to simplify the design of the demodulator [1]. Uplink communication (from the implanted device to the external transmitter) is usually achieved by modulating the load of the implanted inductor and detecting the effects of such modulation on the current flowing through the external inductor (Load Shift Keying - LSK). Thus, no implantable RF transmitter is used.

\footnotetext{
* Corresponding author. Tel.: +41 21693 8169; fax: +41 216930909.

E-mail address: jacopo.olivo@epfl.ch (J. Olivo).
}

Remote powering through inductive link has been studied since several decades and it has already reached the market with commercial products [2]. Nevertheless, the miniaturization of the implanted inductors, while preserving power efficiency, is still an open research topic. Different solutions have been proposed in the literature to realize implantable inductors: litz-wire coils $[3,4]$, printed spiral inductors [5], and micro-fabricated inductors are the most common approaches.

\subsection{Inductor micro-fabrication}

Micro-fabrication can sensibly decrease the size of implantable coils, thus reducing the discomfort of the patient. Indeed, the receiving inductors can be fabricated directly on the substrate hosting the system to be powered. The main challenge of this approach is to preserve the inductor quality factor while dealing with small structures. To that aim, trace thickness should be in the order of tenths of micrometers to reduce the resistance and increase the quality factor.

Several solutions have been proposed in the literature. Thick electroplated inductors on SU8 flexible substrate [6] and silicon $[7,8]$ have been proposed. In these works, the coil thickness does not exceed $24 \mu \mathrm{m}$. Ref. [9] has shown an inlaid process to fabricate electroplated inductors on a silicon substrate with a trace thickness of $60 \mu \mathrm{m}$. However, the proposed inlaid technique cannot be directly used on substrates other than silicon.

Copper electroplating is a well-known technique. Ref. [10] describes the fabrication of thick inductors by using different types of photoresist. EPON SU8, AZ4562, and AZ9260 are considered 


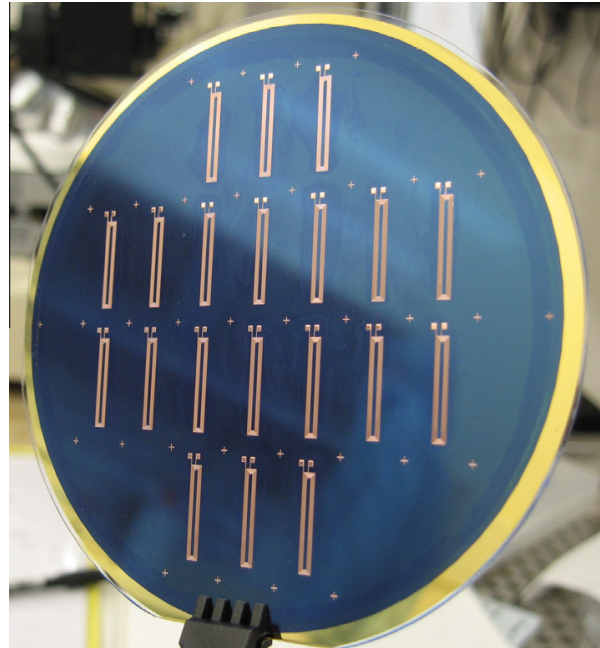

Fig. 1. Micro-fabricated spiral inductors during the fabrication process (step (c) of Fig. 5 - Section 3). The blue layer surrounding the inductors is Ordyl Alpha960. (For interpretation of the references to colour in this figure caption, the reader is referred to the web version of this article.)

due to the possibility to obtain high-thickness mold. AZ4562 and AZ9260 enable trace thickness up to $92 \mu \mathrm{m}$ with high aspect ratios, up to $6: 1$. However, they require multi-layer deposition of photoresist. EPON SU8 enables high trace thickness (in the order of $100 \mu \mathrm{m}$ ) with a single-layer deposition. However, the poor adhesion of SU8 with the copper seed layer and the difficulty to remove it after the electroplating discourage its use.

In this paper, the use of Ordyl Alpha960 is explored to obtain high-thickness traces with single-layer deposition (Fig. 1). Ordyl is a dry film, negative photoresist designed to be developed and stripped in mildly alkaline solutions. This feature enables its stripping without damaging the electroplated copper structures. Moreover, it offers good resistance to leaching during electroplating. Finally, its flexibility is useful during all the process steps, especially those involving mechanical polishing.

The micro-fabricated inductors have been designed to power the implantable system proposed in [11]. That system consists of an integrated sensor embedded on a silicon substrate, together with micro-fabricated electrodes, to perform electrochemical measurements of human metabolites, such as glucose and lactate. The system, designed to be implanted in the subcutaneous tissues of the body, has a maximum power consumption of $540 \mu \mathrm{W}$ [12]. It bidirectionally communicates with the external transmitter by means of ASK modulation (downlink up to $100 \mathrm{kbps}$ ) and LSK modulation (uplink up to $66.6 \mathrm{kbps}$ ). Micro-fabrication of the receiving inductor directly on the silicon substrate hosting the sensor can noticeably decrease the total volume of the system, as compared with the case where multi-layer printed inductors are used [5].

The remainder of the paper is organized as follows. In Section 2, different geometries are simulated for the receiving inductor to define a convenient geometry. In Section 3, the fabrication process flow is described. The performance of the fabricated inductors is shown in Section 4. Finally, Section 5 concludes the paper.

\section{Inductor design}

In this section, several geometries are analyzed for the receiving inductor. These geometries are then compared in terms of quality factor. The quality factor $Q$ of an inductor can be generally defined as

$Q=\frac{\llbracket \mathrm{m}\left\{Z_{\text {in }}\right\}}{\mathbb{R e}\left\{Z_{\text {in }}\right\}}$, where $Z_{\text {in }}$ is the input impedance of the coil. If the inductor operates fairly below the self-resonant frequency, the quality factor can be approximated as

$Q=\frac{\omega L}{R}$

where $L$ is the inductance value, $R$ is the resistance value, and $\omega$ is the working angular frequency. The quality factors of the transmitting and the receiving inductors directly affect the link efficiency $\eta$, defined as the ratio between the power dissipated on the load and the total dissipated power [13]:

$\eta=\frac{k^{2} Q_{1} Q_{2} \frac{R_{2}}{R_{L}}}{\left(1+\frac{R_{2}}{R_{L}}+k^{2} Q_{1} Q_{2} \frac{R_{2}}{R_{L}}\right)\left(1+\frac{R_{2}}{R_{L}}\right)}$,

where $Q_{1}$ and $Q_{2}$ are the quality factors of the transmitting and the receiving inductors, respectively, $k$ is the coupling coefficient, $R_{2}$ is the resistance of the receiving inductor, and $R_{L}$ is the load resistance.

The simulations reported in this section are performed with the commercial electromagnetic solver Sonnet. All the inductors are simulated as a thick layer of copper onto a substrate of $525 \mu \mathrm{m}$ silicon $+1 \mu \mathrm{m}$ silicon dioxide. The area of the receiving inductor is set to $14.88 \times 2 \mathrm{~mm}^{2}$ (Fig. 2). This constraint is determined by the size of the silicon substrate that will host the micro-fabricated coils [11]. Since the inductive link described in [11] operates at $5 \mathrm{MHz}$, the comparison of the different geometries is performed at that frequency. The quality factors reported in the simulations are obtained by using Eq. (1).

\subsection{Tracethickness}

A first set of simulations is performed to check the relation between the quality factor and the trace thickness. The results are shown in Fig. 3. As reported in the Introduction, a higher trace thickness involves a lower resistance, thus increasing the quality factor. However, due to skin and proximity effects, the quality factor cannot be continuously increased by increasing the trace thickness. In this case, a thickness higher than $60 \mu \mathrm{m}$ does not lead to significant improvements in terms of quality factor.

The thickness of the micro-fabricated inductors is set to $60 \mu \mathrm{m}$. This value corresponds to the maximum thickness achievable by using a single layer of Ordyl Alpha960 in the micro-fabrication

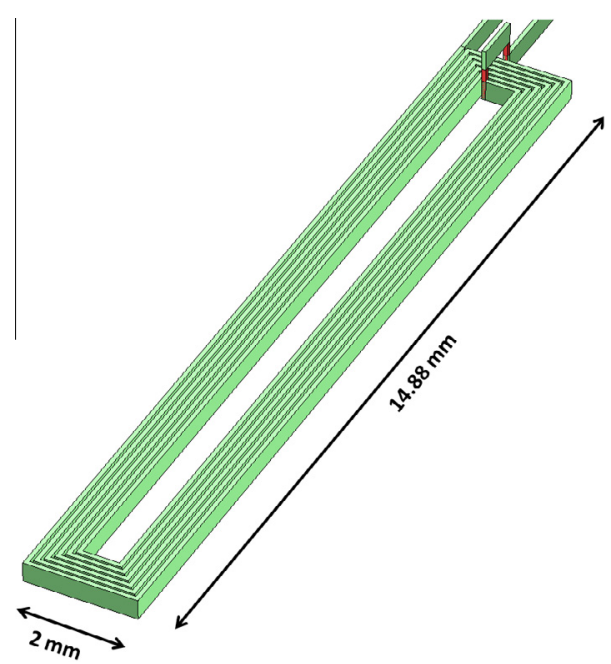

Fig. 2. The inductors, having a size of $14.88 \times 2 \mathrm{~mm}^{2}$, are simulated onto a substrate of $525 \mu \mathrm{m}$ silicon $+1 \mu \mathrm{m}$ silicon dioxide. 


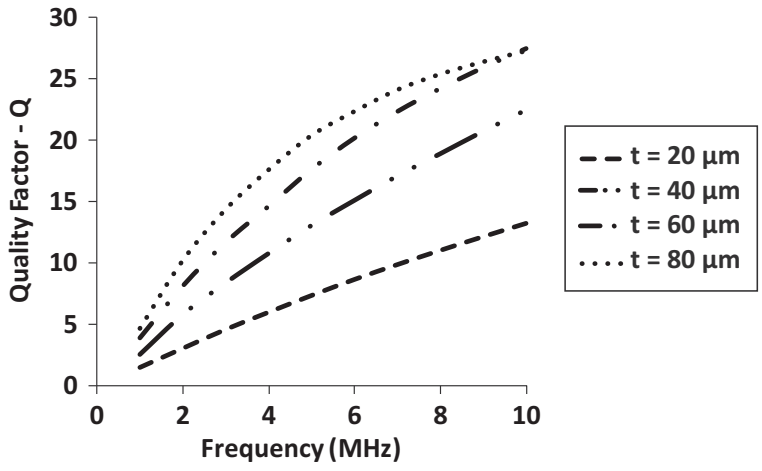

Fig. 3. A higher trace thickness $t$ decreases the inductor resistance, thus increasing the quality factor.

process. A higher trace thickness is compatible with the fabrication process, although it requires more than one layer of photoresist.

\subsection{Number of turns}

A second set of simulations is performed to determine the number of turns of the spiral inductor. The results of these simulations are shown in Fig. 4. The quality factor is weakly dependent on the number of turns, for a given shape and size of the coil. This is in agreement with the constant-Q assumption $[13,14]$. This assumption considers the quality factor of a coil independent of the number of turns, for a given shape and size of the coil.

Coherently with the simulation results, the number of turns is fixed equal to 6 (Fig. 2). The trace width is equal to $80 \mu \mathrm{m}$ and the inter-trace width is equal to $40 \mu \mathrm{m}$.

\section{Fabrication process flow}

The inductors are fabricated on $525 \mu \mathrm{m}$ thick, $100 \mathrm{~mm}$ silicon $\langle 100\rangle$ wafers. These substrates are p-type doped and exhibit a resistivity of $0.1-100 \Omega \mathrm{cm}$. Wafers are delivered with $1 \mu \mathrm{m}$ wet oxide on the top layer; the oxidation is used to insulate the inductors from the silicon substrate.

Two thin layers of chromium $(20 \mathrm{~nm})$ and gold $(100 \mathrm{~nm})$ are evaporated by Joule effect on the wafer top layer (Leybold - Optics LAB600H) (Fig. 5a). Chromium layer is used for adhesion; gold layer is used in the next steps as a conductive seed layer for copper electroplating. Similarly to SU8, Ordyl exhibits poor adhesion with copper. For this reason, a chromium-gold seed layer is used instead of copper.

A layer of $60 \mu \mathrm{m}$ Ordyl dry film (Alpha960 - Elga Europe) is laminated on the wafers. Ordyl dry film is a negative photoresist designed to be developed and stripped in mildly alkaline solutions.

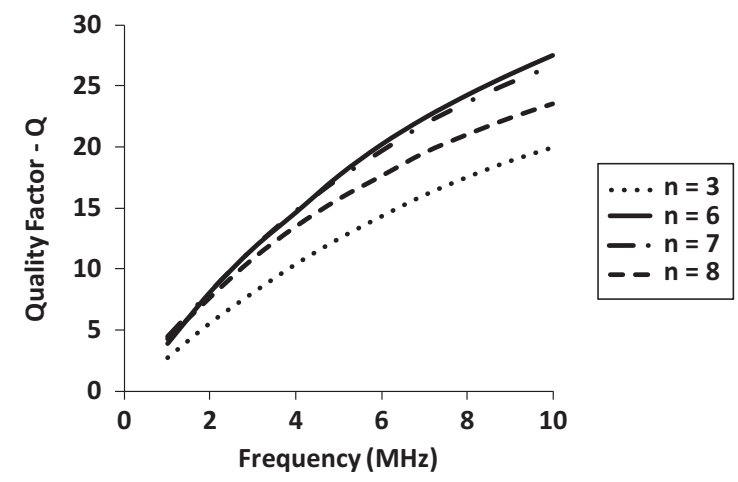

Fig. 4. The quality factor, for a given shape and size of the spiral inductor, is weakly dependent by the number $n$ of turns.

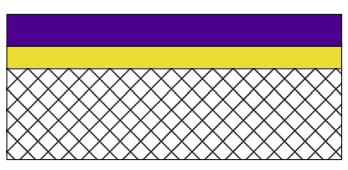

(a)

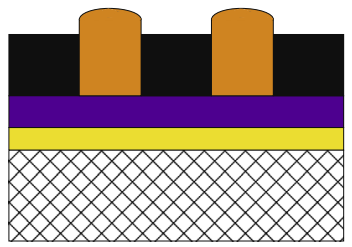

(c)

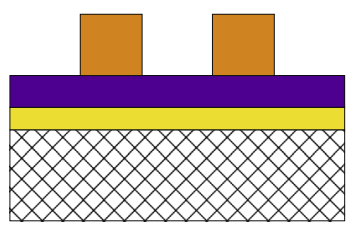

(e)

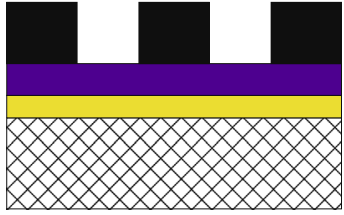

(b)

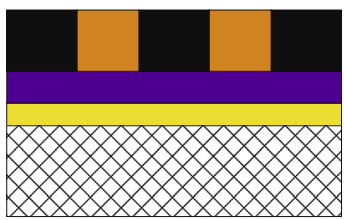

(d)

(f)

Copper
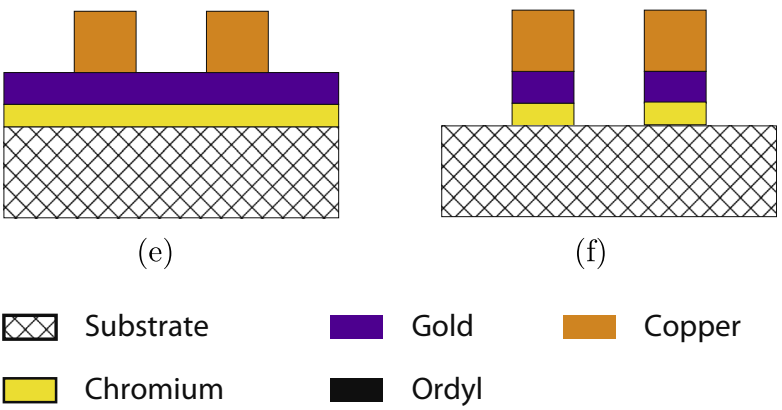

Fig. 5. Fabrication process flow. (a) Seed layer evaporation; (b) photolithography; (c) electroplating; (d) polishing; (e) stripping; (f) wet etching.

The lamination process is performed at $110^{\circ} \mathrm{C}$ (PhotoPro33 Laminator). Wafers are pre-heated prior the lamination to dehumidify.

Trenches are opened in the Ordyl film to enable copper electroplating of the inductor traces (Fig. 5b). To that aim, photoresist is exposed to UV light source (Süss MicroTec - MJB4) having a power density of $15 \mathrm{~mW} / \mathrm{cm}^{2}$. Exposure time is set to $6 \mathrm{~s}$. Photoresist developing is performed at room temperature in sodium carbonate $\left(\mathrm{Na}_{2} \mathrm{CO}_{3}, 0.8-1.2 \%\right)$ for a time period between $270 \mathrm{~s}$ and $330 \mathrm{~s}$. To remove organic residuals at the bottom of the trenches, plasma cleaning is performed after developing (power: $200 \mathrm{~W}$; pressure: $50 \mathrm{~Pa} ; \mathrm{O}_{2}$ flow: $200 \mathrm{sccm}$; time: $20 \mathrm{~s}$ ).

Copper is then electroplated inside the trenches (Fig. 5c). Wafers are merged in a copper plating bath (Rohm \& Haas - Copper Intervia 8510). The exposed gold layer acts as a cathode. A direct current of $130 \mathrm{~mA}$ is forced through it. The current density, defined as the ratio between the cathodic current and the area of the exposed gold layer, is $40 \mathrm{~mA} / \mathrm{cm}^{2}$. Electroplating is performed for $1 \mathrm{~h}$ and $30 \mathrm{~min}$.

Electroplating is not-uniform along the wafer area. Differences in thickness of several micrometers are measured between different inductors; differences in thickness are also measured between different traces of the same inductor. To achieve a uniform thickness of $60 \mu \mathrm{m}$, copper traces are polished until they are planar with the film surface (Fig. 5.d). To that aim, chemical-mechanical polishing is performed (STEAG - Mecapol E460) (slurry: 1609BTA; head speed: $45 \mathrm{rpm}$; plate speed: $50 \mathrm{rpm}$; pressure: $34 \mathrm{kPa}$; time: $25 \mathrm{~min}$ ).

Photoresist is then stripped with sodium hydroxide $(\mathrm{NaOH}$, $2.5 \%$ ) at room temperature (Fig. 5e). The stripping solution swells the Ordyl film; as a consequence, the film is peeled off from the surface. However, stripping of Ordyl between the inductor traces is difficult, since the film tends to get stuck in the narrow intertrace spaces when swelled. To increase the stripping temperature does not lead to noticeable improvements and can damage the copper by creating a layer of copper(II) hydroxide $\left(\mathrm{Cu}(\mathrm{OH})_{2}\right)$ 


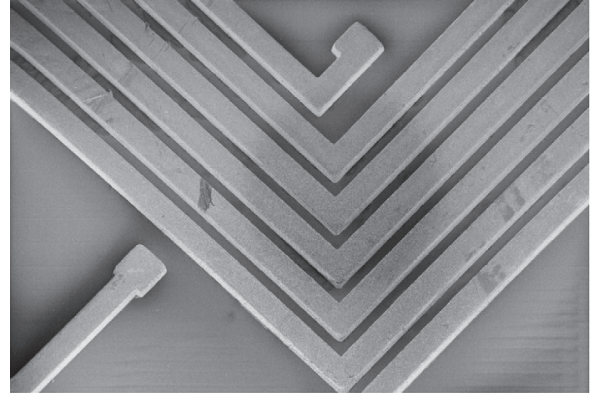

(a)

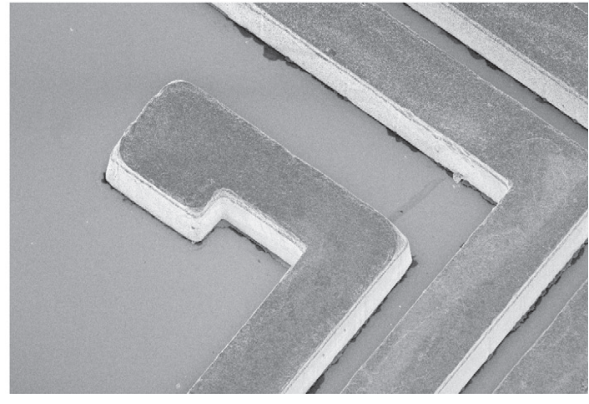

(b)

Fig. 6. SEM pictures of the micro-fabricated inductors.

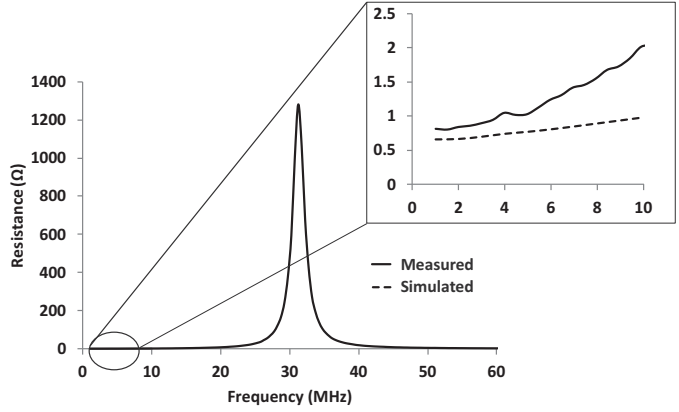

(a) Resistance

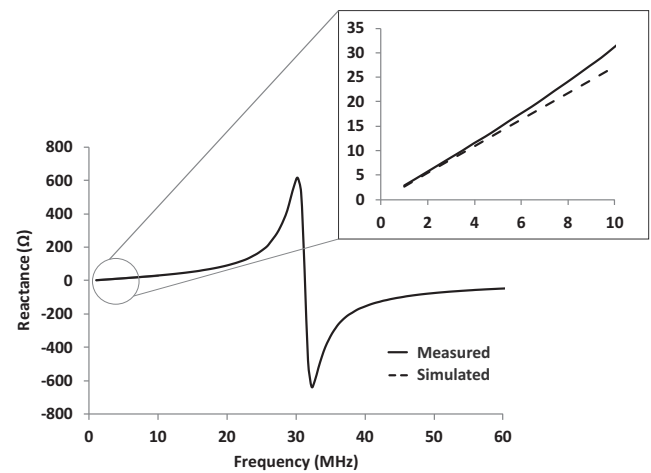

(b) Reactance

Fig. 7. Electrical characteristic of the micro-fabricated inductors. Measurements show a good agreement with the simulations.

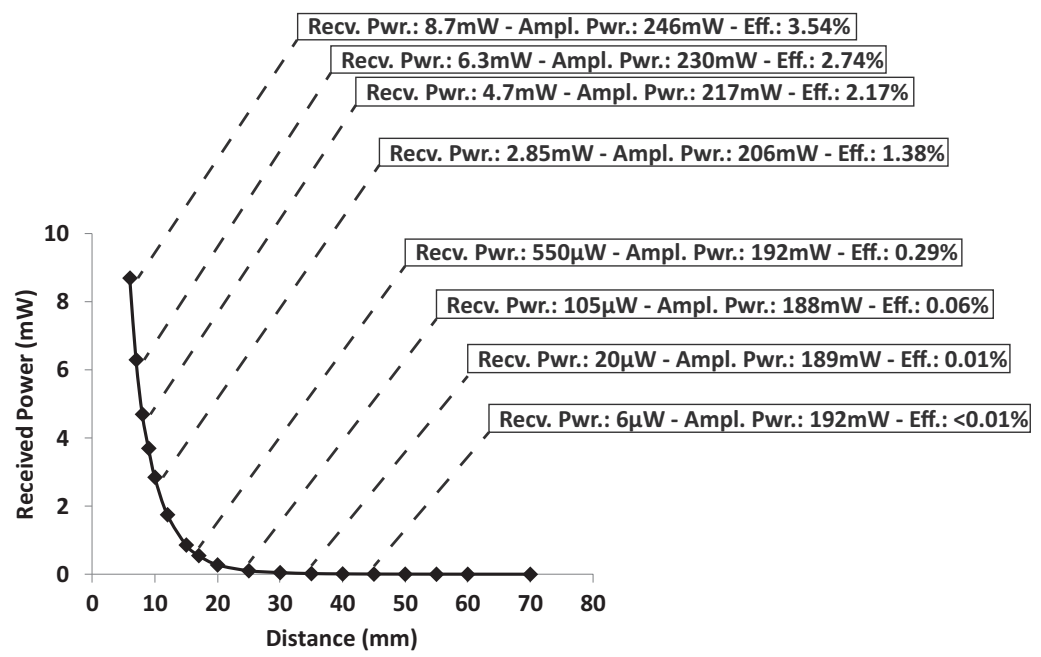

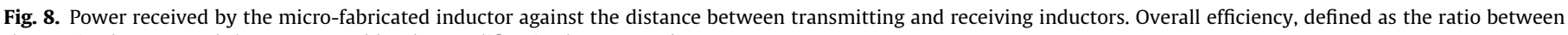
the received power and the power used by the amplifier, is also reported.

around the traces. To improve the film removal, the stripping solution is stirred during the process and a pipette is used to agitate the solution in proximity of the inductor trenches, thus easing the stripping process.

Once the film removed, the inductor traces are still short-circuited due to the conductive chromium-gold seed layer (Fig. 5e). To conclude the process, those two metals are removed from the inter-trace spaces by wet etching (Fig. $5 \mathrm{f}$ ).

Gold is removed by using $\mathrm{KI}+\mathrm{I}_{2}(25 \mathrm{~g} / \mathrm{L}$ and $12 \mathrm{~g} / \mathrm{L}$, respectively) at room temperature. The etch rate is between $40 \mathrm{~nm} / \mathrm{min}$ and $80 \mathrm{~nm} / \mathrm{min}$; however, several minutes are needed to completely remove the gold layer between the traces. The gold etcher reacts with the copper by creating a thick layer of copper(I) iodide (CuI) around the traces; that layer can be removed by using ammonia $\left(\mathrm{NH}_{3}, 45 \%\right)$, thus exposing again the copper traces.

Chromium is removed by using $\mathrm{KMnO}_{4}+\mathrm{Na}_{3} \mathrm{PO}_{4}(60 \mathrm{~g} / \mathrm{L}$ and $200 \mathrm{~g} / \mathrm{L}$, respectively) at room temperature. The etch rate is about $40 \mathrm{~nm} / \mathrm{min}$ but, similarly to what happens with gold, several minutes are needed to completely remove the chromium between the traces. Pictures of the inductors, captured with a scanning electron microscope (SEM) at the end of the fabrication process, are shown in Fig. 6. 


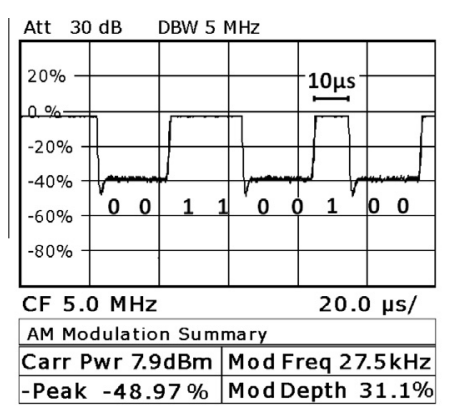

(a) Downlink communication

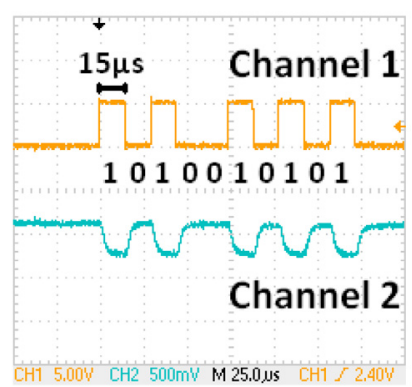

(b) Uplink communication

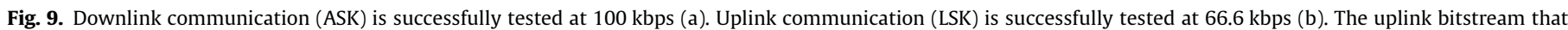
short-circuits the internal inductor is reported on channel 1 . The bitstream received on the external transmitter is reported on channel 2.

Finally, wafers are cut to separate the inductors and aluminum wire bonding is used to connect the inner edge of every inductor to the contact pad laying outside the spiral. Epoxide resin is applied over the bonding area to prevent short-circuits.

\section{Measurements}

Micro-fabricated inductors are tested with a network analyzer (Rohde \& Schwarz - ZVL) to check the electrical characteristic. The measured values of resistance and reactance are reported in Fig. 7. The comparison with the simulated values, also reported in Fig. 7, shows a good agreement between measurements and simulations. At the working frequency $(5 \mathrm{MHz})$, the error on the resistance is in the order of few hundreds milliohms, while the error on the inductance is in the order of tenths of nanohenries.

The self-resonant frequency is located at $31.3 \mathrm{MHz}$. At $5 \mathrm{MHz}$, the inductance value is $0.46 \mu \mathrm{H}$.This value is similar to that obtained at lower frequency. Indeed, at $500 \mathrm{kHz}$ the inductance is equal to $0.45 \mu \mathrm{H}$. At $5 \mathrm{MHz}$, the quality factor calculated by using Eq. (1) is equal to 13.65. Eq. (2) returns 13.36.

Micro-fabricated inductors are tested within a real setup to measure the link efficiency. Power is sent to the receiving inductors by means of the IronIC patch [15]. That patch is equipped with a high efficiency class-E power amplifier driving a 30-turn, $38 \times 24 \mathrm{~mm}^{2}$ spiral inductor. The received power against the distance between transmitting and receiving inductors is reported in Fig. 8. The received power is measured by using a spectrum analyzer (Rohde \& Schwarz - ZVL) connected to the receiving inductor. A purely capacitive matching network is used between the instrument and the receiving coil to maximize the received power.

A maximum received power of $8.7 \mathrm{~mW}$ is measured within a distance of $6 \mathrm{~mm}$ between the inductors. This value is sufficient to power the target device [12]. At $6 \mathrm{~mm}$, the link efficiency, as calculated in Eq. (3), is equal to 25\%. In Fig. 8 is also reported the overall efficiency, defined as the ratio between the received power and the power dissipated by the external class-E amplifier driving the transmitting inductor. At $6 \mathrm{~mm}$, the overall efficiency is equal to 3.54\%. This performance is comparable to that reported in [5], where multi-layer spiral inductors on PCB are used within the same setup.

Finally, data communication is tested. Downlink communication is tested up to $100 \mathrm{kbps}$ by modulating the amplitude of the power carrier generated by the transmitter (ASK). The modulating bitstream is detected on the receiving inductor by using an amplitude demodulator (Rohde \& Schwarz - ZVL). In Fig. 9a is shown the received bitstream. Uplink communication is tested up to $66.6 \mathrm{kbps}$ by short-circuiting the receiving inductor according to the outgoing bitstream (LSK). This load modulation is detected by monitoring the supply current of the external power amplifier [15]. When the receiving coil is short-circuited, the amplifier supply current decreases. In Fig. 9b, the uplink bitstream detected by the external transmitter (channel 2) is shown together with the uplink bitstream acting on the receiving inductor (channel 1).

\section{Conclusions}

A micro-fabrication process is proposed to realize high-thickness spiral inductors for the remote powering of implantable biosensors through inductive link. According to the given constraints, several geometries are simulated and compared. Ordyl Alpha960 dry film is used to obtain high-thickness structures by using a single-layer deposition. Finally, a $60 \mu \mathrm{m}$ thick, 6-turn spiral inductor is realized on silicon. The micro-fabricated coil is measured and tested within a real setup. When powered with the IronIC patch, the inductor receives up to $8.7 \mathrm{~mW}$ within a distance of $6 \mathrm{~mm}$ between the transmitting and the receiving coils. At that distance, the link efficiency is equal to $25 \%$. The overall efficiency of the link, that takes into account the power dissipated by the external amplifier, is equal to $3.54 \%$. This performance is comparable to that obtained in previous works, where multi-layer spiral inductors realized on PCB were used. Finally, downlink communication (ASK) has been successfully tested at $100 \mathrm{kbps}$ and uplink communication (LSK) has been successfully tested at $66.6 \mathrm{kbps}$.

\section{Acknowledgements}

The research work presented in this paper was funded by the iIronIC project and by the NanoSys project. The NanoSys project is within the program ERC-2009-AdG-246810, the i-IronIC project was financed with a grant from the Swiss Nano-Tera.ch initiative and evaluated by the Swiss National Science Foundation.

J. Olivo thanks the CMi Staff of EPFL for the assistance during the fabrication process. A special thanks to A. Guillet, from CMi, for the precious suggestions during the micro-fabrication process and to M. De Marchi, from LSI-EPFL, for the useful discussions.

\section{References}

[1] J. Olivo, S. Carrara, G. De Micheli, IEEE Sens. J. 11 (7) (2010) 1573-1586.

[2] Activa RC Recharging System 37751.

[3] G. Kendir, W. Liu, G. Wang, M. Sivaprakasam, R. Bashirullah, M. Humayun, J Weiland, IEEE Trans. Circuits Syst. I Reg. Papers 52 (2005) 857-866.

[4] B. Lenaerts, R. Puers, Biosens. Bioelectron. 22 (2007) 1390-1395.

[5] J. Olivo, S. Carrara, G. De Micheli, IEEE Trans. Biomed. Circuits Syst. 7 (2013) 536-547.

[6] P. Basset, A. Kaiser, D. Collard, L. Buchaillot, J. Vac. Sci. Technol. B 20 (2002) 1465-1470.

[7] Y. Choi, J. Yoon, IEEE Electr. Device L 25 (2004) 76-79. 
[8] R. Puers, G. Vandevoorde, D. De Bruyker, J. Micromech. Microeng. 10 (2000) 124-129.

[9] J. Wu, V. Quinn, G. Bernstein, J. Micromech. Microeng. 14 (2004) 576-586.

[10] M. Brunet, T. OD́onnell, J. OB́rien, P. McCloskey, S. Mathuna, J. Micromech. Microeng. 12 (2002) 444-449.

[11] S. Carrara, S. Ghoreishizadeh, J. Olivo, I. Taurino, C. Baj-Rossi, A. Cavallini, M. Op de Beeck, C. Dehollain, W. Burleson, F. Moussy, A. Guiseppi-Elie, G. De Micheli, Sensors 12 (2012) 11013-11060.
[12] S. Ghoreishizadeh, S. Carrara, G. De Micheli, in: IEEE BioCAS Conf. (2011) 460463.

[13] B. Lenaerts, R. Puers, Omnidirectional Inductive Powering for Biomedical Implants, Springer, 2009.

[14] W. Ko, S. Liang, C. Fung, Med. Biol. Eng. Comput. 15 (1977) 634-640.

[15] J. Olivo, S. Carrara, G. De Micheli, in: IEEE I2MTC Conf. (2012) 286-289. 